\title{
A Study on the Implications of COVID-19 Pandemic on Families with Autism Spectrum Disorder
}

\author{
Menezes Ida Sylvia ${ }^{1 *}$, K. T. Shwetha ${ }^{2} \& D^{\prime}$ Mello Laveena ${ }^{3}$ \\ $1^{*}$ Research Scholar, College of Social Sciences and Humanities, Srinivas University, Mangalore \\ Orcid ID: 0000-0001-7654-9217; Email: dishaasd2006@gmail.com \\ ${ }^{2}$ Assistant Professor, School of Social Sciences \& Humanities, Srinivas University, Mangalore, \\ Karnataka, India. \\ Orcid ID: 0000-0003-0895-6333; Email: kt.shwetha@gmail.com \\ ${ }^{3}$ Associate Professor, School of Social Sciences \& Humanities, Srinivas University, Mangalore, \\ Karnataka, India. \\ Orcid ID: 0000-003-1935002X; Email: lavynoronha@gmail.com
}

Area/Section: Health Sciences.

Type of the Paper: Research Case Study.

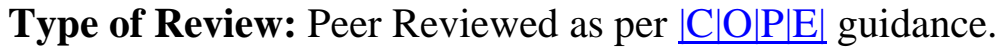

Indexed in: OpenAIRE.

DOI: https://doi.org/10.5281/zenodo. 5726922

Google Scholar Citation: IJHSP

\section{How to Cite this Paper:}

Sylvia, Menezes Ida., Shwetha, K. T., \& D’Mello, Laveena, (2021). A Study on the Implications of COVID-19 Pandemic on Families with Autism Spectrum Disorder. International Journal of Health Sciences and Pharmacy (IJHSP), 5(2), 28-41. DOI: https://doi.org/10.5281/zenodo.5726922

International Journal of Health Sciences and Pharmacy (IJHSP)

A Refereed International Journal of Srinivas University, India.

Crossref DOI : https://doi.org/10.47992/IJHSP.2581.6411.0072

(C) With Author.

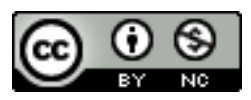

This work is licensed under a Creative Commons Attribution-Non-Commercial 4.0 International License subject to proper citation to the publication source of the work.

Disclaimer: The scholarly papers as reviewed and published by the Srinivas Publications (S.P.), India are the views and opinions of their respective authors and are not the views or opinions of the SP. The SP disclaims of any harm or loss caused due to the published content to any party. 


\title{
A Study on the Implications of COVID-19 Pandemic on Families with Autism Spectrum Disorder
}

\author{
Menezes Ida Sylvia ${ }^{1 *}$, K. T. Shwetha ${ }^{2} \&$ D'Mello Laveena ${ }^{3}$ \\ $1^{*}$ Research Scholar, College of Social Sciences and Humanities, Srinivas University, Mangalore \\ Orcid ID: 0000-0001-7654-9217; Email: dishaasd2006@ gmail.com \\ ${ }^{2}$ Assistant Professor, School of Social Sciences \& Humanities, Srinivas University, Mangalore, \\ Karnataka, India. \\ Orcid ID: 0000-0003-0895-6333; Email: kt.shwetha@gmail.com \\ ${ }^{3}$ Associate Professor, School of Social Sciences \& Humanities, Srinivas University, Mangalore, \\ Karnataka, India. \\ Orcid ID: 0000-003-1935002X; Email: lavynoronha@gmail.com
}

\begin{abstract}
Purpose: In the exceptional disruption and social isolation of the COVID-19 pandemic, households round the arena are confronted with questions of ways their youngsters can thrive in those conditions. The ever-present demanding situations for kids with unique fitness needs such as autism spectrum disorder (ASD), the disaster relay particular problems due to terrible influences of the COVID-19 pandemic.

Methodology: A sample of 30 parents of children with ASD has been included in the study during the second wave of the pandemic. Parents have been asked to fill out self-report questionnaire through Google form.

Findings: Researcher has tried to understand the vulnerability of ASD individuals, the associated co-morbidity, challenges encountered, turmoil on daily routine, unreached therapeutic modalities and the stressors. The role of healthcare facilities during the pandemic like COVID -19, the measures taken to ease the burden of ASD community has been studied. The benefit of online services and tele-health during pandemic along with its significance and positive benefits has been identified. It is the best medium of practice for people with limited resources trying to reach efficient behavioural health professionals through technology. Interstate and international opinions and differential diagnosis always remained incomplete as people have no resources to reach the best, pandemic has strengthened the connectivity and collaboration to individuals in need and made it possible.
\end{abstract}

Originality: The efforts to prioritize the need of the vulnerable community and their responsibilities in reintegrating them into society with risk measures have been weighed. The impact on the overall growth and development and on the psychological realm has been identified with its outcome and the management. What best can be offered and done during the relevant crisis and what measures need to be taken has been deeply observed.

Paper type: Research Case Study.

Keywords: COVID-19 pandemic, Autism spectrum disorder, Vulnerability, Challenges, Stressors, Online services, Social isolation.

\section{INTRODUCTION :}

The sudden outbreak of novel coronavirus (COVID-19) has caused uncertainty in all aspects of society resulting in universal fear, anxiety leading to stress. The unanticipated pandemic provoked challenges to cope with the rapid changes that forced adaptability in the survival of the fitness [1]. If we retrieve the pandemic history of any infectious disease, it highlights the need to secure the psychological and behavioral domains, as they are in the list of most vulnerable domains due to adverse effects. The unavoidable mental stress and provoked anxiety, sleep disturbances, insecurity due to the negative impact of the pandemic are the frontline stressors [2]. The fear of unknown end will further add up the existing adverse effects raising the question of till when? Most importantly, the bar graph pattern of the infection precisely caused terrified fear in individuals 
preventing them from leading a normal routine life. The due course of the spread of infection affected the overall members of the family and children with their innocent understanding, the most affected. The fortunate essence of children safety inconsideration with COVID-19, in the early spread has reduced and the burden of getting infections with vulnerability raised as we progressed with the delta plus variant [3, 4]. Though a thoughts of us are not alone, the whole world is facing and suffering from the pandemic, the challenges to face the consequences of suspension of education, due to quarantine and lock downs, the closure of social gatherings, healthcare facilities, jeopardize the development of the children. Across community it has been crucial to engross swiftly changing health services to accommodate daily living during the ongoing pandemic. The psychological factors such as fear of death, losing loved ones in this pandemic, along with the consequences of autistic features, have accelerated the psychological disorders significantly; anxiety disorder in the forefront due to the unpredictable future. In comparison to the normal population, the autism spectrum disorder people have inordinate anxiety due to the unknown concept of pandemic and difficulty to understand the myth concealing the reality [5]. The pervasiveness of pandemic, the interruptions in forthcoming happenings disrupts the organized routine, bringing chaos and stress in the general population increasing struggles due to its unpredictability. The limitation of the adaptation of the new events due to its unknown prediction and shifts in life, turmoil the living in autism population rather more challenging.

\section{REVIEW OF LITERATURE :}

\subsection{Increased vulnerability and autism:}

Vulnerability is the level to which an individual or population is unable to predict, adapt, tolerate and recuperate from the outcomes of disaster [6]. As families around the world are suffering and facing with the question of how best to support their children, the drastic condition arises how to support children with autism as they are cognitively weak and not in the picture of understanding or comprehending the word around them. Pandemic has been a challenge to already challenged kids with autism [7-9]. The foremost challenge is, how to introduce the whole scenario of COVID! As the abstract concepts are restricted in the brains of these little ones, panic behind the spread of the disease, deadly outcome of the COVID, the consequences of not wearing the mask or the need of social distancing is the highly challenging task that anyone would dare to carry forward to reduce the misbehaving. Their cognitive executive functions and intellectual levels curtail their ability to comprehend and to carry on the warnings issued on COVID-19 infection [10]. An overwhelming question arises in the mind of the researcher, what is more vulnerable? The consequences or outcome of the protocols of the pandemic in its prevention in the life of autism? As far as the disease condition concerned the autism diagnosis is the vulnerability itself in the life of children with autism. The additional vulnerability of pandemic adverse consequences makes it more vulnerable due to the constraints imposed in accordance with its prevention. The life without the routine ongoing schedules in the life of autism children unremarkable. The child on the spectrum says it all, the severity of the illness, destroys the laid foundation, of behavioral, educational, vocational, recreational, social and self-schedules. The strategies applied in the prevention protocol of pandemic impediments all the hardships and dedication inculcated in raising a lotus from the pond. The measures to be taken to challenge the consequences of the pandemic deterrents in the well-being of these neuro-developmentally challenging children prioritize the parents and healthcare professional's agenda. Impact of the pandemic in already diagnosed autism children though falls in one extreme of the spectrum, the other end focuses on the undiagnosed children between the age of 18 to 24 months as the developmental stage, early age provokes the symptoms of autism and leads to the missing of the diagnosis due to the lockdown and quarantine. The delay in completing assessments and diagnosing the child due to the closure of healthcare facilities as initial screening has been interrupted due to sudden lock down. Delayed diagnosis procures delayed treatment. The early intervention protocols and the only hope of erasing the unpredictable severity of the disruptive characteristics of the diagnostic mask the outcome. The critical development periods if used intelligently, in the diagnosis and treatment schedules can signify the outcome. The delay in the diagnosis ultimately leads to delayed treatment aggravating cognitive functions, emotional outcomes, behavioral components as the behavior cannot be identified, its purpose of occurrence due to the inability to communicate as the language disorder says it all. The closure of the medical care facilities and the interventions and therapy centers has led to the postponement of the screening of the children with early recognized characteristics of autism and the pervasive developmental disorders [11-12]. The outcome led to delayed treatments and therapeutic modules worsening the behavioral functions and cognitive skills [13]. 


\subsection{Autism Spectrum Disorder:}

Autism Spectrum Disorder is the umbrella term and always exists with the comorbid illness unless it is a classical type. The comorbid features are remarkable! ASD co-occurs with physical disabilities, epilepsy, obsessive compulsion disorders, and cerebral palsy, which increase the risk of severe complications from COVID-19 [14]. These hallmark features negatively impacted by the COVID-19 pandemic in ASD put them at greater risk. When we excurse the characteristics of autism, the core features of this heavily labeled diagnosis are the social communication and the functional language challenges. Children diagnosed children with autism under therapeutic intervention initiating to communicate and this unpredicted pandemic strategy of lockdown and closure of all therapy centers and social gatherings at the right time devouring the buds of speech and language as they erupt! devouring the buds of speech and language as they erupt! Being one of the major adverse effects was further complicated by wearing masks and further confusing the distorted signals of communication in the brain, blocking them by wearing masks during socialization. What irony of these kids at the time of learning to pronounce or to repeat a word or sentence! Considering this single outcome in the acquisition of language in the life of autism children, we can imagine or concretely calculate the outcome of disruptive behavior and cognitive skill development, comprehensive and intellectual skills as well as the attention increment or focus. The empathy is that COVID pandemic is definitely not for families of children with autism prevalence, raising the question of what further devastate the life of these families? When we discuss about the vulnerability of various factors influencing these vulnerabilities and ethnicity is one such factor that affects health disparities leading to devastation [15-16]. The medical access and services significantly contribute to the disparity along with its genetic and biological susceptibility among the normal population who raised in autism community [17]. As described by the core feature of autism, social communication and interaction, further deteriorates due to immediate paralysis of supportive environment due to the pandemic restrictions. The social developments through neighbors, peer groups, nurseries and therapy care as individual and small group interactions, gently challenges the social isolation making impossible with direct interactions and involvement. While the core features are having the direct impact due to unpredicted pandemic period, how would anyone manage to carry on the diagnosis along with pandemic strategies? The inherent differences in executive functioning of learning new skills, basic needs adaption, persistent learning, managing self-control, working on emotions, make it difficult to follow and master [18]. The root cause of receptive and expressive language difficulties produces emotional disturbances that lead to disruptive behavior [19]. The inability to understand the cause of the behavior along with uncontrolled emotions further exaggerates the challenges, making it impossible to manage. The catastrophic outcome is the inability to explain or make them to understand the utter need to break the schedules, unavoidable circumstances of sticking inside one room or in house due to the devastating influence of corona as well as the observation of symptoms of the covid19 illness. The challenge of effectively and efficiently communicating the pain, addressing the symptoms of the illness and coldness of emotions due to the expressive communication challenges creates distress in the life of child with autism [20,21]. The only golden rule for maintaining a calm atmosphere is the adherent routine schedules. The routines are more predictable to an autism child, and maintains a balanced environment to both the diagnosed child and the family. The unpredictability of COVID-19 shuttered such balance leading to distress due to the uncertainties of pandemic.

\subsection{Mental Health:}

What makes an individual with ASD to be more affected in his mental health? As discussed above, the chore characteristics of autism affecting its behavioral component significantly due to inability to communicate or comprehend the built-up emotions bring about more aggression, disruptive behavior in simple words as tantrums and the refusal or avoidance of engaging in any daily engagements. The only way to handle these behaviors and to get normalcy in these kids is to engage them in physical activities and routines to drain the tempo to reduce hyperactivity. Unfortunately, the home quarantine and lockdown sealed this availability making them impossible to carry on. The unmet calming effect due to the indoor and outdoor activities has disrupted the regulating effect. As talk about the mental health, what actual bothers the physical health of an individual with autism, irrespective of the age? Why the individuals with autism suffer from poor health compared to the typical group? Studies have shown that individuals with autism face sensory impairments or sensory sensitivities [22]. The sensitivities may be over responsiveness or responsiveness to various stimuli as each autism has uniqueness. Inconsideration to five major sensory organs and stimulations, food sensitivity is one of the major issues, which indulge in the health component, the interference of which leads to poor 
health. Personal aversion toward food and the picky eating habits exacerbates the situation during a pandemic due to unavailability of the supply of the demanding items as well as the shutdown of the major stores. The compulsion on the usage of mask adds to existing irritability due to sensory issues, making it impossible to rationalize it. Children with sensitivities find it too difficult to wear a mask as the tags and even the elastic ties too sensitive and are impossible to bear with. These are some possibilities why the autism population has a higher risk of poor health compared to the normal population [23].

\subsection{Autism with comorbidity:}

The comorbidity with autism complicates the skills required for adaptation, learning and a healthy lifestyle. Children with intellectual disabilities and physical disabilities struggle in executive functioning skills as well as ambulatory skills, respectively. Co-occurring illness such as epilepsy with its treatment effects interferes in learning and attention span. Same as in the case of psychotic illnesses. The drug therapy, which is mandatory and pervasive interferes in the stimulation functions as part of the therapeutic modules. Obsessive compulsive disorders on the other hand are unavoidable comorbid disorders, which restrict all therapeutic outcomes due to its added characteristics and drug regimes. This comorbidity intensifies the vulnerability toward psychological, emotional and physical deprivation in this ongoing pandemic [24]. In conditions such as developmental disabilities, the whole concept of progressive damage that occurs during the developmental stages anchors the mental health of individuals challenging them to adapt, acute stress-related situations such as post-traumatic or COVID-19 pandemics as present scenarios [25]. Increasing acute psychotic or mood disorders with suicidal ideation or tendencies, due to anticipated fear of loss of loved ones and trouble adapting [26]. The pervasive developmental disordered people have increased vulnerability to mental health disorders than to the typical population [27]. The unknown end of COVID-19 pandemic and the co-occurring complexities such as emotional disorders decay resilience [19]. What makes the individuals with autism more vulnerable toward the adverse consequences of COVID-19 is the question to be answered. As research has shown its heightened superiority in investigating the etiological factors or risks of susceptibility of infectious disorders in individuals with ASD, are probable to share genetic and physiological risk factors. Proinflammatory cytokines play a major role in escalating the inflammatory process as studies have documented [28]. Cytokine storm can lead to upregulate the phenotype of COVID-19 infection leading to death due to cardiac and lung damage [29]. As discussed, pro-inflammatory tendency inclines the risk of optimization of symptoms in case of contamination [30]. The comorbid condition such as type 2 diabetes predispose poor recovery. As emerging research suggests that the vulnerability and susceptibility of contaminating the virus are high in populations with comorbid disorders such as diabetes mellitus and hypertension, as well as poor outcome, people with autism have strong predisposition due to genetic and physiologic risk factors [31-32].

\section{METHODOLOGY :}

This study proposes to use a cross-sectional exploratory survey design by recruiting an available purposive sample of 30 parents of children with autism spectrum disorder in India. The objectives of the study are; to study the vulnerability of autism children; to study the impact due to mitigation strategies of COVID-19; to study the impact on educational challenges; to study the impact on vocational challenges; to study the impact on educational challenges; to study the impact on economic challenges; to study the impact on psychological health; to study the impact on behavioral health services.

\section{ANALYSIS :}

\subsection{Impact due to mitigation strategies of COVID-19:}

The unpredicted mitigation strategies to battle COVID - 19 considerably affected the families of autism. The stay-at-home remedies and social distancing-handicapped individuals with autism drastically compared with other disabilities. It's like fish out of water without access to these critically needed services and supports. The outcome of these strategies considerably affected each and everyone in the cycle, right from the child with autism, to the parents, family members, caregivers and professionals, resulting in even greater vulnerability. Also, the multiple service providers for educational, vocational and skills-oriented need providers had affected vice versa [31]. Children and adults with ASD struggle the most due to in situ learning interruption as the generalization of the task and skills serves the best in autism children [22]. The practical teaching and learning methods as best taught in the environment that best suits the goals to be achieved were shuttered due to the lock down restrictions and social distancing. These individuals had to learn basic skills, 
community skills, educational and vocational skills under one roof with limited resources. Both the caregiver and environment had to be remodified and trained to accommodate the new learning strategies. Though it affected the whole population on the same swing, the autism population critically suffered due to the inability to understand the COVID climax, the reorganization or rescheduling of the daily activities, locking under one premise, without knowing the reason as well as the mental confusion and chaos. The helplessness of making the best use of the available resources with the adaptability is the thought to concern. Professionals had to modify their services or interrupt, due to the pandemic leading to limitation of the availability. Applied behavioral therapy (ABA) serves best in understanding the cause of any challenging behaviors, its attention seeking or escapism. Through which the underlying purpose will be identified thereby appropriate measures are taken either to minimize or restrict through extinction. ABA is intensive that provides actionable steps through individualized program. There is no one size fits all phenomena hence a regular intensity depending on the age group and the level of ASD needed for the better outcome. Usually, 3 to 4 hours a day six days per week therapy scheduled for the demanding needs of social functional communication, academic needs depending on the abilities and mastering the learned activity through highly reinforcing the positive behavior. There are no untaught or self-learned phenomena as observational learning in ASD. Each and every action, behavior or a situation need to be taught including giving back or fighting for one's own right or even slapping when needed. All encouraging behaviors need to be reinforced positively and disruptive actions reinforced through negative reinforcement such as time out or withdrawing the stimulus. As we understand the core features of autism or ASD are social communication, the stay-at-home strategies have completely locked lifestyle and learning in autism individuals. Naturalistic interventions through social communication intervened due to social distancing protocols. And wearing masks further closes the mouth of a minimal communicating person, making it impossible to initiate any communication. These logistical barriers in the journey of ABA therapies and autism individual further challenges integrated therapies such as speech and language and or the occupational. The small classroom settings best applied in the immediate care of the autism child as the one -to- one attention therapy faded, traversing them into the main stream. The closure of the therapy centres or the small integrated classroom environments not continues at home settings due to social distancing. Online teaching or online therapy modules either of ABA or of any speech language or occupational therapies will not be effective as virtual learning will not be applied in real situations when there are challenges of attention as well as the transferring skills. Moreover, Autism Spectrum Disorder is one such disability that requires specialized therapeutic interventions and specialized centres for services provided by specialists [32]. Furthermore, limited resources and work at home or out of work parents during this pandemic, encounter economic barriers to engage in therapies as they are out of reach for many families. Economic constraints deepen the barriers between these two groups with and without adequate resources such as premises, infrastructures, internet availability, computer and electronic gadgets suitable for home setting.

\subsection{Impact on Educational challenges:}

Does education really in children with autism? Here, the age group specification favors the outcome of teaching or learning at the early age. As we always say and advice and witness the early intervention intrusions and its fruitful outcomes. The plasticity of brain intensifies due to persistent interventions, making it possible to learn if started right on time. And that is the reason for concern why education need to be given an utmost importance in the life of autism? The shutdown of all educational institutions in the beginning of COVID pandemic to mitigate the spread of infection panicked many families both typically developing and with disabilities. There was no room for any planning or developing modalities to fit in distance learning. It was highly challenging to adapt to the new curriculum that fits in for any population in society. The challenge was admirable when complex communication needs to be met and the curriculum to be developed for various disabilities, including ASD due to its uniqueness. As the pandemic pave to adapt to the new life style, educational services with limited resources emerged and distance learning played an important role in the field of education [32]. Distance learning raised the psychological well-being of the many variant groups of population, including the parents. It's just out of the question to imagine the idleness of each developmental age group and its physical emotional psychological consequences. Though the structured environment and the schedules were not feasible, the level of perseverance raised some degree of learning. The challenge with the educational qualification of the parents took the stand, as the special educator's role best played by the parents specially the mothers, fulfilling the demanding chores of household as well as work from home in case of work-life balancing mothers. In the midst of ongoing preferred activities, children with autism had to attain and sustain their attention, resisting the temptations, controlling hyperactivity, tolerating the sensory 
issues. It is totally a mess if resources such as proper structured infrastructure, a big spacious room without disturbances to sustain attention as well as the devotion of the required number of hours toward scheduled teaching, the ambience required and support from other members of the family are inadequate. The use of electronic gadgets for ongoing online teaching had to be more professionally applied and the equipment used as fun filled medium of entertainment with apps had to change their role in imparting knowledge and learning. Definitely that is one of the major challenges in mothers to control irritability and the temper tantrums as well as the mode of communication as listening and hands on. However, inexperienced parents should give their best in learning new skills as well as required training to effectively implement the education plans. Effective use of delivering reinforcements, controlling the distractions and navigating the tantrums is rather herculean. Adaptation to new learning is challenging even in the life of parents depending on the age with other demands.

\subsection{Impact on Vocational Challenges:}

Irrespective of the age group individual affected with autism as pervasive developmental disorder face significant challenges, including vocational. Adults with ASD face loss of community services due to the pandemic [32]. With the essence of social isolation due to social communication disorder the adult autism individuals face greater potential risk than typical adults [33]. They isolate themselves from social gatherings and community activities [34], due to their disability they are less employed and restricted with their participation in employment-oriented tasks [35]. They have unique lifestyle, well-structured and organized, requiring attention and accompaniment for security and transport reason, when employment concerned. Sudden changes in their schedules due to stay-at-home and lock downs they face adjustment problems to the newly provoked situation, which is unstructured and time oriented. Proper usage of task and the time pass will be highly challenging as they will not be able to engage in ready to use leisure activities. Most of the time they are helpless not knowing how to use the available resources due to functional fixedness. Autism adults have unique lifestyle that builds confidence and independence, with constant support. Most them live in residential care groups with vocational services that define their life style. A sudden outbreak of COVID as infectious, forced shut down of this residential care with social distancing protocols that created turmoil in these adults and family members. The risk of contamination required maximum health guidelines to combat COVID as well as challenged social isolation [36-37]. Family members had to readjust to the temporaryshuffled situation with the daily living and the time management of both the disabled person and them with great sacrifices. Adaptation is part and parcel of every human being and we adapt sooner or later subjected to the degree of adjustments. But in the life of autism, is rather unpredictable as the disorder itself cumbersome.

\subsection{Impact on Economic Challenges:}

The initial math of pandemic brought considerable anxiety and fear globally to re-join the premises once we all were much attached with and adjusted. The first fear of contamination of the COVID-19 virus from our own colleagues and the question of how safe we are at work place! This made the economic situation further degrade leading to severe stress in each and every bread winner of the family. It was of highly challenging decision-making ability of an individual to select the best among the worst. With the extreme fear of carrying the virus at home and contaminating the loved ones and losing them, which was a nightmare in most extended families. In the life of autism, it was and yet rather more adversely challenging as the individual had to compromise with the short duration of living with the stay-at-home diplomacy and again moving back to the community for school or work or vocational trainings [38]. The rigidity with adaptation in autism critically damages mental hygiene, and rescheduling with readaptation with the fear of temporary fallback, makes no sense in these individuals with cognitive skills deficits. Though gradual reintroduction to the social environment is the criteria for a better adjustment, regression of skills once mastered was challenging due to the lack of practice for months together as well as the fine-gross motor co-ordination. Retraining and mastering once learned skills, copying with wearing of mask, and most restricting strategy of social distancing These adults with autism suffer significant economic hardships equally or greater than the other population.

\subsection{Impact on Psychological Health:}

Psychological health gets affected mainly due to anxiety caused by direct and indirect impact of various stressors along with depression as it pent up and not catharized. As discussed, the stress due to the unpredictable outbreak of corona pandemic that shattered the daily living of people, especially children and 
adults with autism. The sudden closure of critically essential intervention services devastated the already affected autism community. The unpreparedness and untrained parents face a maximum catastrophe. Special educators and therapists play minimal roles as physical contacts were out of the question due to contamination and the restricted strategies. As well understood, the role of specialists in preparing parents to reach their kids with autism needs independently most of the time not met. It is either due to the recently diagnosed children, who have received the diagnosis and still in the process of understanding and learning how to live with autism kids or due to the work-life balance, they are unable to self-attend the needs of autism individual's or due to the availability of the facilities when financial resources met. Hence, depending on the therapists and caregivers instead of self-preparedness in taking care of the autism individual. Covid19 pandemic as the great teacher to many such parents who are not self-reliant and heavily depend on others. It is like learning the lesson that money can't buy everything and provide everything. The restricted strategies of COVID pandemic as social distancing and lockdown made it impossible to avail them. In spite of various reasons, the need to manage everyday life with autism is highly challenging due to the disruptive behavior and adaptation problems [9,39-40]. Disruptive behavior mainly due to struggle with executive functioning, as they find it extremely difficult to plan and organize themselves to a sudden change, task initiation is highly challenging and self-monitoring. They require constant monitoring and supervision as they lack creativity [17]. One of the core characteristics of autism is restricted interests and they get along with their routine scheduled interest. A sudden change in their behavior of fixed interests causes chaos and it's like an outbreak itself leading them to stuck [41]. A balanced structured schedule and time frame, comforts in developing skills and copying accordingly. Adaptation to new circumstances takes much time and self-effort as well as support from others to reallocate with new interests. Social environments, enjoyed by autism individuals, when they are adapted. Any interference will provoke anxiety due to its sudden interruption and time to adapt to a new environment. Internalizing conditions as anxiety and depression were the major risk factors that directed adolescence and adults to the emergency department during the peak of pandemics [42]. Psychological and social support to re-arrange familiar community activities emerges to relieve stress behind this anxiety. The suicidal tendencies were increased drastically in children and adults with autism due to the impact of the pandemic. Restricted movements inside the house, stay-at-home policies, no social environments and routine outings or schedules [43,24]. Irrespective of developmental stage individuals with ASD were subjected to mental health crisis requiring sudden actions of close monitoring and assessments to avoid eventualities. Institution's infection control strategies for admissions and treatments of these eventualities impede and conversion of multi-specialities to intensive COVID care, causing workforce shortage as well as the availability of bed for inpatient services [44]. Careful supervision and utmost care are landmark in psychiatric care, as alcohol-based sanitization potentially be ingested hazards paving more personnel. The immediate outcome will cause alcohol overuse and or tobacco consumption to combat or relieve temporarily the unaccepted eventualities. Bodily pain or psychosomatic symptoms will add to the existing physical and mental problems by increasing the use of medical resources [45-46]. As it is an unprecedented eventuality, the basic needs of the family complicate the affected psychological condition due to economic fallout raised due to unemployment, shifting of residences, loss of the breadwinner, migrating or relocating and the food insecurity [47].

\subsection{Impact on behavioural Health Services:}

Behavioural health is a major concern in the life of autism individuals as well as the family members, which was greatly impacted due to the sudden outbreak of the pandemic. Behavior health services suddenly paused and or quickly converted to distance services as virtual or telehealth. Applied behavior analysis (ABA) is delivered face to face with proportionately close proximity using shared materials. It is an evidence-based treatment in both outpatient and in-home services [48]. The essence of observation of behavior and the reinforcement schedules in virtual mode is beyond the reach. Autism individual knows to throw temper tantrums with their loved ones holding their leads and practically this type of session does not work in front of camera. It works best to few parents who dedicate themselves with the therapies in the progress of the child, taking guidelines and demonstrations and apply as hands-on. The lack of protocols during this type of pandemic with fear of contagiousness affected considerably during the beginning stage of pandemic [49]. Distance education and telehealth were atypical form of ABA, which was shortly replaced with in-person services in spite of potential risks [50]. Some forms of ongoing support were maintained through telehealth services minimizing the exposure and maintaining social distance in verbally communicating population. Outpatient psychiatry and psychologist visits in the form of telehealth to combat the implications of COVID- 
19 though conducted in a later stage, unsatisfactorily meet the physical examination and the observation of the behavior. Children with antipsychotic drugs and other mood disorders as comorbid, were need to be closely monitored periodically through laboratory tests for liver and kidney functions, were not conducted due to the closure of healthcare services [51]. The most fearful concern and the need were the availability of drug and its consequences due to shortage of drug supply as social distancing compelled the closure of all essentials [52].

\section{DISCUSSIONS :}

\subsection{Accepting the new normal:}

Stay-at-home protocols though served as miracle cure for corona spread, social-emotional trauma never been replaced with the community of autism. Though the typical population underwent considerable damage in these domains, the ASD families due to the functional fixedness and the inability to use the available in -situ resources suffered the most. The digital world extremely met the fun and entertainment fields to the general population on the other hand, which made no difference to the autism community. The uncertainties and unpredictable changes created chaos in planning educational and recreational programs affecting the future preparation. Re-engagement with the peer group and companionship of new peer group is highly challenging causing strangers' anxiety as well as accommodation confront. The dilemma of re-engagement to the routines and the return to quarantine as the corona wave spikes, continuous adjustment to uncertainties is stressful to individuals with cognitive dysfunction. The ultimate outcome of temper tantrums leading to exacerbate behavior due to unpredictable environments. The brief transition to the existing condition or the unprecedented dark future, is the worry of many parents as they are confused about how to proceed! The inevitable situations contribute more stress to the parents as they must restart their work- life, in spite of the closure of the school and childcare facilities, appointing new caregivers, raising new stranger anxiety to both the receiver and parents. As we witnessed, the emergency preparedness to situations like COVID -19 pandemic is impractical as no one is ready to risk their own lives, neither the healthcare personnel except in critical care unit. In these situations, vulnerable groups like ASD combating various domains of life without behavioral and psychological support are gruel some.

\subsection{Management of Parental Stress:}

Parental stress mainly due to financial concerns, already existed, which was boosted by the pandemic due to unemployment or decrease in pay cheque. Problem solving skills were degraded due to the unavailability of health services, affecting self-efficacy and self-care. The closure of childcare facilities affected the most to parents under essential care services where they had no room to place their disabled young ones. Understanding the diagnosis with the commitment of working toward its welfare was never fulfilling, which raised the engrain resulting in punishment and losing tempers [53]. Dysfunctional interactions emerged and groomed due to the pandemic situation, which definitely not a positive impact on autism families.

\subsection{Readiness for Major Life Transitions:}

Telehealth and online flat forms have shown remarkable outcome in assisting individuals to accept the new environment, to adjust and adapt to new normal, and to reintegrate in the community. Traversing creative solutions toward resilience and preparedness in near future. Emergency preparedness for optimizing coping, guidance and resilience will be crucial for intervention and mental health hygiene [54]. Though the emergence of digitalization and the online services were in practice in various healthcare sections, before the pandemic, mental health had restrictions due to direct clinical observation and its findings to apply regularly [55]. Pandemic made it possible to avail as the best alternative including vulnerable groups with ASD. It is the best medium of practice for people with limited resources trying to reach efficient behavioral health professionals through technology. Interstate and international opinions and differential diagnosis always remained incomplete as people have no resources to reach the best, pandemic has strengthened the connectivity and collaboration to individuals in need and made it possible.

\section{SIGNIFICANCE OF THE STUDY :}

The study explores the impact of the pandemic on children with autism, their cognitive and intellectual abilities to comprehend the protocols applied, through warnings issued to protect themselves from deadly corona contamination, in preventing the spread through social distancing and home quarantine. The study understands the comprehensive skills toward the vital need of hand washing and wearing a mask in children 
with autism as prevention strategy. The study also attempts to understand the offered structured strategies and guided special education if any, while handling autism therapeutic modules, to help parents of children with autism during COVID-19 pandemic. Such modalities will help develop and enhance support programs and interventions in the near future, considering the extensions of COVID-19.

\section{CONCLUSION :}

The question of significance of vulnerability arises in autism spectrum disorder community about any pandemic including the COVID-19, as they have social interaction and functional language limitations. The pandemic worsens the characteristics due to prolonging social isolation. What best been offered to these individuals with ASD is the concern and how suitably we make it possible without further worsening the tag of vulnerability! Prioritizing the reintegration to these groups ensures a reduction in post-pandemic impact. Social environments such as schools can be converted into intervention and training centres during this type of pandemic to accommodate individuals with ASD strictly following the social distance and curfews. Collaboration with local community leaders in instrumenting the crisis in ASD families and providing such an environment for the upliftment of individuals with ASD. COVID-19 has made to realize the extent of preparedness measures during similar crisis for productive and effective skills management. Potentials to strengthen these services at the local level, to meet the unique needs in the future. Critical innovations, including the individual with ASD in the process when applicable, to prepare measures will empower solely. Online resources have made remarkable contribution to the problem-solving skills of ASD communities, hence the availability of electronic equipment's to the unaffordable families would be utmost important to provide structured supportive programs.

\section{REFERENCES :}

[1] Baweja, R., Brown, S.L., Edwards, E. M. (2021). COVID-19 Pandemic and Impact on Patients with Autism Spectrum Disorder. Journal of Autism Developmental Disorder, 29(1), 4950-4959.

Google Scholar 7 CrossRef $\ngtr$

[2] Bellomo, T. R., Prasad, S., Munzer, T., \& Laventhal, N. (2020). The impact of the COVID-19 pandemic on children with autism spectrum disorders. Journal of Paediatric Rehabilitation Medicine, 13(3), 349354.

Google Scholar $7 \quad$ CrossRef $x^{7}$

[3] Cox, D. J., Plavnick, J. B., Brodhead, M. T. (2020). A Proposed Process for Risk Mitigation During the COVID-19 Pandemic. Behaviour Analysis in Practice, 13(2), 1-7.

Google Scholar $x^{\top} \quad$ CrossRef $x^{\top}$

[4] Song, W., Li, J., Zou, N., Guan, W., Pan, J., Xu, W. (2020). Clinical features of paediatric patients with coronavirus disease (COVID19). Journal of Clinical Virology, 127(1), 1-7.

Google Scholar $x^{7} \quad$ CrossRef $x^{\top}$

[5] Van Steensel, F. J., Bögels, S. M., \& Perrin, S. (2011). Anxiety disorders in children and adolescents with autistic spectrum disorders: a meta-analysis. Clinical child and family psychology review, 14(3), 302317.

Google Scholar $x^{7} \quad$ CrossRef $x$

[6] World Health Organization. (2002). The world health report 2002: Reducing risks, promoting healthy life. World Health Organization, 1-26.

Google Scholar $x^{\top} \quad$ CrossRef ${ }^{\top}$

[7] Den, Houting, J. (2020). Stepping Out of Isolation: Autistic People and COVID-19. Autism in Adulthood. E-pub ahead of print. 2(1), 103-105.

Google Scholar $x^{\prime} \quad$ CrossRef $x^{7}$

[8] Liu, J. J., Bao, Y., Huang, X., Shi, J., Lu, L. (2020). Mental health considerations for children quarantined because of COVID-19. Lancet Child Adolescent Health, 4(5), 347-349.

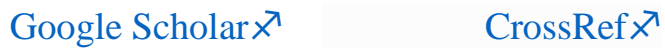


[9] Singh, S., Roy, D., Sinha, K., Parveen, S., Sharma, G., Joshi, G. (2020). Impact of COVID-19 and lockdown on mental health of children and adolescents: A narrative review with recommendations, Psychiatry Research, 293(1), 1-11.

Google Scholar X

CrossRef $x^{7}$

[10] Courtenay, K., Perera, B. (2020). COVID-19 and people with intellectual disability: Impacts of a pandemic. Irish Journal of Psychological Medicine, 37(1), 231-236.

Google Scholar $x^{\top}$

CrossRef $x^{\top}$

[11] McPheeters M., L.., Weitlauf, A., Vehorn, A., Taylor, C., Sathe, N., A., Krishnaswami, S. (2015). Screening for Autism Spectrum Disorder in Young Children: A Systematic Evidence Review for the US.. Preventive Services Task Force. Epub ahead of print, 13 (1), 691-696.

Google Scholar \

CrossRef $x^{7}$

[12] Zwaigenbaum, L., Bauman, M., L., Fein, D., Pierce, K., Buie, T., Davis, P., A. (2015). Early screening of autism spectrum disorder: Recommendations for practice and research. Pediatrics, 136(1), S60-81. Google Scholar $x^{\top}$

CrossRef $x^{\top}$

[13] Neumeyer, A. M., Anixt, J., Chan, J., Perrin, J. M., Murray, D., Coury, D. L. (2019). Identifying Associations Among Co-Occurring Medical Conditions in Children with Autism Spectrum Disorders. Academic Pediatrics, 19(3), 300-306.

Google Scholar X'

CrossRef $x^{\top}$

[14] Macias Gil, R., Marcelin, J. R., Zuniga-Blanco, B., Marquez, C., Mathew, T., \& Piggott, D. A. (2020). COVID-19 pandemic: disparate health impact on the Hispanic/Latinx population in the United States. The Journal of infectious diseases, 222(10), 1592-1595.

Google Scholar 7 CrossRef 7

[15] Raisi-Estabragh, Z., McCracken, C., Bethell, M. S., Cooper, J., Cooper, C., Caulfield, M. J., ... \& Petersen, S. E. (2020). Greater risk of severe COVID-19 in Black, Asian and Minority Ethnic populations is not explained by cardiometabolic, socioeconomic or behavioural factors, or by $25(\mathrm{OH})$ vitamin D status: study of 1326 cases from the UK Biobank. Journal of Public Health, 42(3), 451-460. Google Scholar $x^{\top}$

CrossRef $x^{7}$

[16] Anderson, D. K., Lord, C., Risi, S., DiLavore, P. S., Shulman, C., Thurm, A., \& Pickles, A. (2017). American Psychiatric Association. (2013). Diagnostic and statistical manual of mental disorders. Washington, DC: Author. The Linguistic and Cognitive Effects of Bilingualism on Children with Autism Spectrum Disorders, 21(1), 1-197.

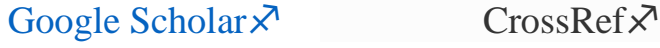

[17] Wallace, G. L., Kenworthy, L., Pugliese, C. E., Popal, H. S., White, E. I., Brodsky, E. (2016). Realworld executive functions in adults with autism spectrum disorder: Profiles of impairment and associations with adaptive functioning and co-morbid anxiety and depression. Journal of Autism and Developmental Disorders, 46(3), 1071-1083.

Google Scholar $x^{7} \quad$ CrossRef $x^{7}$

[18] Hubbard, K., \& Trauner, D. A. (2007). Intonation and emotion in autistic spectrum disorders. Journal of Psycholinguistic Research, 36(2), 159-173.

Google Scholar $x^{\top} \quad$ CrossRef $x^{\top}$

[19] Rattaz, C., Dubois, A., Michelon, C., Viellard, M., Poinso, F., \& Baghdadli, A. (2013). How do children with autism spectrum disorders express pain? A comparison with developmentally delayed and typically developing children. PAIN®, 154(10), 2007-2013.

Google Scholar ${ }^{7} \quad$ CrossRef $x^{\top}$

[20] Kinnear, D., Rydzewska, E., Dunn, K., Hughes-McCormack, L., Melville, C., Henderson, A. (2020). The relative influence of intellectual disabilities and autism on sensory impairments and physical disability: A whole-country cohort of 5.3 million children and adults. Journal of Applied Research in Intellectual Disabilities, 33(5),1059-1068.

Google Scholar $7 \quad$ CrossRef 7 
[21] Dunn, K., Rydzewska, E., Macintyre, C., Rintoul, J., \& Cooper, S., A. (2019). The prevalence and general health status of people with intellectual disabilities and autism co-occurring together: A total population study. Journal of Intellectual Disability Research, 63(4), 277-285.

Google Scholar $x^{7} \quad$ CrossRef $x^{7}$

[22] Dotson, W. H., Leaf, J. B., Sheldon, J. B., \& Sherman, J. A. (2010). Group teaching of conversational skills to adolescents on the autism spectrum. Research in Autism Spectrum Disorders, 4(2), 199-209. Google Scholar $x^{\top} \quad$ CrossRef $x^{7}$

[23] Liu, N., Zhang, F., Wei, C., Jia, Y., Shang, Z., Sun, L. (2020). Prevalence and predictors of PTSS during COVID-19 outbreak in China hardest-hit areas: Gender differences matter. Psychiatry Research, 287(1), $1-7$.

Google Scholar $x^{7} \quad$ CrossRef $メ$

[24] Ammerman, B. A., Burke, T. A., Jacobucci, R., \& McClure, K. (2020). Preliminary investigation of the association between COVID-19 and suicidal thoughts and behaviours in the US. Journal of Psychiatric Research, 134(1), 32-38.

Google Scholar $x^{\top} \quad$ CrossRef $x^{\lambda}$

[25] Diaz, A., D., \& Baweja, R. (2021). The role of neurotropism in psychiatric patients with COVID-19. European Archives of Psychiatry and Clinical Neuroscience, 271(1), 385-386. Google Scholar 7 CrossRef 7

[26] Saghazadeh, A., Ataeinia, B., Keynejad, K., Abdolalizadeh, A., Hirbod-Mobarakeh, A., \& Rezaei, N. (2019). A meta-analysis of proinflammatory cytokines in autism spectrum disorders: Effects of age, gender, and latitude. Journal of Psychiatric Research, 115(1), 90-102.

Google Scholar $x^{\top} \quad$ CrossRef $x^{\top}$

[27] Lin, S. H., Zhao, Y. S., Zhou, D. X., Zhou, F. C., \& Xu, F. (2020). Coronavirus disease 2019 (COVID19): Cytokine storms, hyper-inflammatory phenotypes, and acute respiratory distress syndrome. Genes \& Diseases, 7(4), 520-527.

Google Scholar $x^{7} \quad$ CrossRef $x^{7}$

[28] de Sousa Lima, M. E., Barros, L. C., M., \& Aragão, G. F. (2020). Could autism spectrum disorders be a risk factor for COVID-19? Medical Hypotheses, 144(1), 1-4.

Google Scholar $\nearrow^{\top} \quad$ CrossRef $x^{\top}$

[29] Cariou, B., Hadjadj, S., Wargny, M., Pichelin, M., Al-Salameh, A., Allix, I. (2020). Phenotypic characteristics and prognosis of inpatients with COVID-19 and diabetes: the CORONADO study. Diabetologia, 63(1),1500-1515.

Google Scholar ${ }^{7} \quad$ CrossRef $x^{7}$

[30] Chen, M. H., Lan, W. H., Hsu, J. W., Huang, K. L., Su, T. P., Li, C. T. (2016). Risk of developing type 2 diabetes in adolescents and young adults with autism spectrum disorder: A nationwide longitudinal study. Diabetes Care, 39(5), 788-793.

Google Scholar $\nearrow^{\top} \quad$ CrossRef ${ }^{7}$

[31] Cidav, Z., Lawer, L., Marcus, S. C., \& Mandell, D. S. (2013). Age related variation in health service use and associated expenditures among children with autism. Journal of Autism and Developmental Disorders, 43(4), 924-931.

Google Scholar $x^{\top}$

CrossRef $x^{\top}$

[32] Eshraghi, A. A., Li, C., Alessandri, M., Messinger, D. S., Eshraghi, R. S., Mittal, R., \& Armstrong, F. D. (2020). COVID-19: Overcoming the challenges faced by individuals with autism and their families. The Lancet Psychiatry, 7(5), 481-483.

Google Scholar $7 \quad$ CrossRef 7

[33] Howlin, P., Moss, P., Savage, S., \& Rutter, M. (2013). Social outcomes in mid-to later adulthood among individuals diagnosed with autism and average nonverbal IQ as children. Journal of the American Academy of Child \& Adolescent Psychiatry, 52(6), 572-581.

Google Scholar ${ }^{7} \quad$ CrossRef $x^{7}$ 
[34] Tint, A., Maughan, A. L., \& Weiss, J. A. (2017). Community participation of youth with intellectual disability and autism spectrum disorder. Journal of Intellectual Disability Research, 61(2), 168-180.

Google Scholar メ

CrossRef $x^{\top}$

[35] Roux, A. M., Shattuck, P. T., Cooper, B. P., Anderson, K. A., Wagner, M., \& Narendorf, S. C. (2013). Postsecondary employment experiences among young adults with an autism spectrum disorder. Journal of the American Academy of Child \& Adolescent Psychiatry, 52(9), 931-939.

Google Scholar $x^{\top} \quad$ CrossRef $x^{\top}$

[36] Giallonardo, V., Sampogna, G., Del Vecchio, V., Luciano, M., Albert, U., Carmassi, C. (2020). The impact of quarantine and physical distancing following COVID-19 on mental health: Study protocol of a multicentric Italian population trial. Frontiers in Psychiatry, 11(1), 1-10.

Google Scholar $x^{7} \quad$ CrossRef $x^{7}$

[37] Constantino, J. N., Sahin, M., Piven, J., Rodgers, R., \& Tschida, J. (2020). The impact of COVID-19 on individuals with intellectual and developmental disabilities: Clinical and scientific priorities. American Journal of Psychiatry, 177(11), 1091-1093.

Google Scholar ${ }^{7} \quad$ CrossRef 7

[38] Neece, C., McIntyre, L. L., \& Fenning, R. (2020). Examining the impact of COVID-19 in ethnically diverse families with young children with intellectual and developmental disabilities. Journal of Intellectual Disability Research, 64(10), 739-749.
Google Scholar $x^{\top}$
CrossRef $x^{7}$

[39] Zwaigenbaum, L., Bauman, M. L., Fein, D., Pierce, K., Buie, T., Davis, P. A. (2015). Early screening of autism spectrum disorder: Recommendations for practice and research. Pediatrics, 136(1), 41-59.

Google Scholar \ CrossRef X

[40] Zwaigenbaum, L., Bauman, M. L., Choueiri, R., Kasari, C., Carter, A., Granpeesheh, D (2015). Early Intervention for children with autism spectrum disorder under 3 years of age: Recommendations for practice and research. Pediatrics, 136(1), 60-81.

Google Scholar ${ }^{\top} \quad$ CrossRef $x^{\top}$

[41] Lam, K., Bodfsh, J., \& Pivan, J. (2008). Evidence for three subtypes of repetitive behaviour in autism that differ in familiarity and association with other symptoms. Journal of Child Psychology and Psychiatry, 49(1), 1193-1200.

Google Scholar $7 \quad$ CrossRef $\nearrow$

[42] Liu, G., Pearl, A. M., Kong, L., Brown, S. L., Ba, D., Leslie, D. L. (2019). Risk factors for emergency department utilization among adolescents with autism spectrum disorder. Journal of Autism and Developmental Disorders, 49(11), 4455-4467.

Google Scholar $7^{7} \quad$ CrossRef $\chi^{7}$

[43] Mayes, S. D., Calhoun, S. L., Baweja, R., \& Mahr, F. (2015). Suicide ideation and attempts in children with psychiatric disorders and typical development. Crisis: The Journal of Crisis Intervention and Suicide Prevention, 36(1),55.

Google Scholar $x^{7} \quad$ CrossRef $x^{7}$

[44] Li, L. (2020). Challenges and priorities in responding to COVID19 in inpatient psychiatry. Psychiatric Services, 71(6), 624-626.

Google Scholar 7 CrossRef 7

[45] Brooks, S. K., Webster, R. K., Smith, L. E., Woodland, L., Wessely, S., Greenberg, N., \& Rubin, G. J. (2020). The psychological impact of quarantine and how to reduce it: rapid review of the evidence. The lancet, 395(10227), 912-920.

Google Scholar $x^{7} \quad$ CrossRef $メ$

[46] Morganstein, J. C., Fullerton, C. S., Ursano, R. J., Donato, D., \& Holloway, H. C. (2017). Pandemics: health care emergencies. Textbook of disaster psychiatry, 270-284.

Google Scholar X 
[47] Food, D. G. E. (2021). Tracking the COVID-19 recession's effects on food, housing, and employment hardships. Centre on Budget and Policy Priorities, 1-20.

Google Scholar $x^{\top} \quad$ CrossRef $x^{\top}$

[48] Roane, H. S., Fisher, W. W., \& Carr, J. E. (2016). Applied behavior analysis as treatment for autism spectrum disorder. The Journal of pediatrics, 175(1), 27-32.

Google Scholar $x^{7} \quad$ CrossRef $x^{\top}$

[49] Kornack, J., Williams, A., L., Johnson, K., A., \& Mendes, E., M. (2020). Reopening the doors to centrebased ABA services: Clinical and safety protocols during COVID-19. Behaviour Analysis in Practice, 13(1), 543-549.

Google Scholar $x^{\top}$

CrossRef $x^{\top}$

[50] Colombo, R. A., Wallace, M., \& Taylor, R. (2020). An essential service decision model for ABA providers during crisis. Behavior Analysis in Practice, 13(1), 306-311.

Google Scholar $x^{\nearrow} \quad$ CrossRef $x^{7}$

[51] Volkmar, F., Siegel, M., Woodbury-Smith, M., King, B., McCracken, J., \& State, M. (2014). Practice parameter for the assessment and treatment of children and adolescents with autism spectrum disorder. Journal of the American Academy of Child \& Adolescent Psychiatry, 53(2), 237-257.

Google Scholar $x^{7} \quad$ CrossRef $x^{7}$

[52] Badreldin, H. A., \& Atallah, B. (2021). Global drug shortages due to COVID-19: impact on patient care and mitigation strategies. Research in Social and Administrative Pharmacy, 17(1), 1946-1949.

Google Scholar $x^{\top}$

CrossRef $x^{\top}$

[53] Myers, K. M., Valentine, J. M., \& Melzer, S. M. (2008). Child and adolescent telepsychiatry: utilization and satisfaction. Telemedicine and e-Health, 14(2), 131-137.

Google Scholar $x^{7} \quad$ CrossRef $x^{7}$

[54] Griffiths, L., Blignault, I., \& Yellowlees, P. (2006). Telemedicine as a means of delivering cognitivebehavioural therapy to rural and remote mental health clients. Journal of Telemedicine and Telecare, 12(3), 136-140.

Google Scholar $x^{7} \quad$ CrossRef $x^{7}$

[55] Torous, J., \& Wykes, T. (2020). Opportunities from the coronavirus disease 2019 pandemic for transforming psychiatric care with telehealth. JAMA psychiatry, 77(12), 1205-1206.

Google Scholar $x^{\nearrow} \quad$ CrossRef $x^{\nearrow}$

$* * * * * * * * * * *$ 